\title{
SALUD, BIENESTAR Y ESTILO DE VIDA DESDE UN ENFOQUE DE GÉNERO
}

\section{Health, well-being and lifestyle from a gender perspective}

María Dolores Blesa Matienzo*
Consultorio Psicológico Privado

Perú

\begin{abstract}
Resumen
El presente artículo trata sobre el tema de la salud desde el punto de vista del bienestar y el estilo de vida, planteamos que el bienestar de las personas se puede lograr a través de nuestra manera de actuar, considerando importante la salud de las mujeres en particular. En la revisión de estudios con respecto al bienestar, se han incluido temas relacionados a la exclusión social, la salud de las mujeres, la medicalización, la relación salud y enfermedad y las diferencias de género que existen en nuestras sociedades.
\end{abstract}

Palabras claves: Salud, Bienestar, Estilo de vida, Género

\begin{abstract}
This article deals with the issue of health from the point of view of well-being and lifestyle, we propose that the well-being of people can be achieved through our way of acting, considering important the health of women in particular. In the review of studies regarding well-being, we have included topics related to social exclusion, women's health, medicalization, the relationship between health and sickness and the gender differences that exist in our societies.
\end{abstract}

Keyword: Health, Well-being, Lifestyle, Gender

\footnotetext{
* Psicóloga lolablesa@yahoo.es

ORCID: https://orcid.org/0000-0002-9178-7763
} 


\section{INTRODUCCIÓN}

Hemos desarrollado esta temática relacionando la salud con el bienestar en primer término y su derivación del concepto de Psicología de la Salud, especialidad que toma en consideración estos términos para describir aspectos sanos del individuo y su interrelación con el medio ambiente. El bienestar se logra con un nivel de vida aceptable, en el cual se satisfacen las necesidades básicas del ser humano, a nivel físico, psicológico y social. Existen índices de medición del nivel de vida y del estilo de vida de las personas, los cuales nos permiten conocer la forma como vivimos. Otra arista del tema a tratar es el del género, y el relevante papel de las mujeres en el área sanitaria y en los temas de Salud.

\section{SALUD Y BIENESTAR}

Es interesante analizar cómo se relacionan estos términos y aluden a un estilo de vida. Al relacionar los conceptos de salud y bienestar encontramos que el concepto de bienestar se refiere a las actitudes y comportamientos que mejoran la calidad de vida y nos ayudan a llegar a un estado de salud óptimo: es aquel proceso activo dirigido a nuestro estilo de vida en todas sus dimensiones. Representa nuestras acciones responsables que permiten el desarrollo de un nivel adecuado de salud general.

El bienestar deseado, entonces, se obtiene mediante hábitos saludables que resultan de una adecuada adaptación e integración de las dimensiones físicas, mental, social, espiritual y emocional a cualquier nivel de salud o enfermedad. Esto implica que puedes experimentar bienestar, ya sea que te encuentres enfermo o saludable. La salud no es perfecta, pero se encuentra dentro de una escala que clasifica a la salud en niveles, como podemos observar en la figura 1 de Donatell, Snow \& Willcox. (citado en Lopategui, Edgar, 2001).

Fig. 1

Continuum de la salud Donatell, Snow \& Willcox (1999).

\section{LA ESCALA CONTINUA DE ENFERMIEDAD A BIENESTAR}

Enfermedades de Enfemmedades Salud Buna Bienestar

Vida o Muerte Menores Promedio Salud Positivo

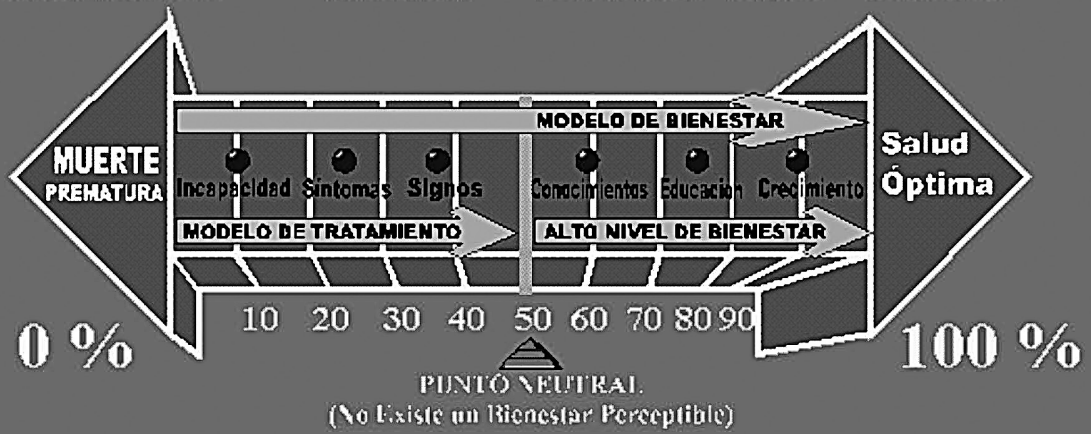

Comportamientos Comportamientos

de Riesgo Saludables 
En la escala Continua o Continuum de la salud, se visualiza que dependiendo del tipo de comportamiento, el nivel de bienestar puede comenzar desde un alto nivel (estilos de vida de alta calidad) hasta enfermedades de vida o muerte (estilos de baja calidad). Este bajo nivel puede, inclusive, ocasionar una muerte prematura (aquella que ocurre antes de los 65 años).

La calidad de vida se refiere al óptimo bienestar entre las cinco dimensiones de la salud (física, mental, emocional, social y espiritual) que caracteriza a ciertos grupos sociales o comunidades.

El concepto de bienestar está relacionado con la calidad de vida de las personas. En general, los indicadores de bienestar pretenden medir el nivel de vida de un grupo específico de la población, tomando en cuenta el carácter multidimensional y complejo de su composición.

Los índices de bienestar se pueden dividir en dos grandes grupos: índices múltiples e índices compuestos.

- Índices múltiples: salud, educación, nutrición, etc.

- Índices compuestos: el ingreso, la ocupación, el acceso a programas sociales y servicios.

Al respecto Sen, A. (citado en Llanos, Martha 2008) concluye que la valoración del bienestar incluye la valoración que hace una persona del modo de vida que ha alcanzado. En ella focaliza su atención en el bienestar y calidad de vida de los seres humanos.

\section{CALIDAD DE VIDA}

Diversos artículos con enfoque de género resaltan el papel de la educación en la superación de barreras de las mujeres, en su cambio de actitudes frente a la maternidad, a la crianza y al trabajo en general, así Rao K. Bhassker (citado en Blesa, María Dolores 1990) refiere que en general cuanto más alto es el nivel de vida y más alto el nivel de educación, más baja es la tasa de natalidad. Un principio general ampliamente reconocido en materia de salud es que en todos los países donde las tasas de natalidad son elevadas, lo son asimismo las tasas de mortalidad materna, perinatal e infantil, por lo que si fuera posible reducir las tasas de natalidad se rebajarían también las de mortalidad. En el mismo sentido Beatriz Merino(1998) resalta que la educación es la escalera que libra a la mujer de los obstáculos originados por las desigualdades de género, pues ejerce una influencia tremenda en el cambio social, el poder internalizar valores como la paz, la tolerancia y el respeto mutuo, además de la necesaria información y conocimiento que promueve; enfatizando que la educación es una inversión que rinde dividendos considerables en materia de crecimiento económico y mejoramiento de la salud y calidad de vida tanto de la mujer como del hombre.

Lamas, Héctor; Lamas, Francisco (2004) sostienen que la forma en que vivimos (estilos de vida) y dónde vivimos (ambiente) son los factores más importantes en determinar los cambios relacionados con el corazón, el sistema inmunológico, los pulmones, los huesos, el cerebro y los riñones.

Luis Oblitas (2008) refiere que "los estilos de vida saludables implican conocer aquellos comportamientos que mejoran o socavan la salud de los individuos. Se entiende por conductas saludables aquellas acciones realizadas por un sujeto, que influyen en la probabilidad de obtener consecuencias físicas y fisiológicas inmediatas y a largo plazo, que repercuten en su bienestar físico y en su longevidad". (p. 17). Señala que en la actualidad, se conoce un número importante de comportamientos relacionados con la salud y son:

\section{Practicar ejercicio físico}

2. Nutrición adecuada

3. Desarrollo de un estilo de vida minimizador de emociones negativas

4. Optimismo, buen humor y risa

5. Adherencia terapéutica

6. Creatividad

Bárbara L. Fredrickson (citada en Fischman, David 2010) ha estudiado las emociones positivas y observó que cuando las personas estaban con emociones positivas, su mente se abría. Cuando 
estamos con emociones positivas, nuestra mente se abre y ampliamos nuestra capacidad de ver el todo.

Cuando el ser humano siente emociones positivas, es más creativo, tiene más capacidad de aprender, ampliar y mejorar sus redes sociales. Otro aspecto que se ha descubierto de las emociones positivas es que nos dan recursos para enfrentar emociones negativas en el futuro. Se ha demostrado que aquellos que tienen más emociones positivas son más resilientes (capaces de recuperarse de la adversidad) y tienen más recursos para superar obstáculos.

Montze Arboix (2013) desarrolla la relación que existe entre estilo de vida y salud. Seguir un estilo de vida saludable aleja multitud de enfermedades como el cáncer, la EPOC o la obesidad, además que mejora la calidad de vida de las personas. Muchos de estos problemas de salud están provocados por factores de riesgo y son modificables, lo que significa que mediante el cambio de hábitos insanos- relacionados con el tabaquismo, dieta y sedentarismo- se podrían reducir la morbilidad y mortalidad asociada.

Al respecto el estilo de vida saludable es un conjunto de patrones de conductas relacionadas con la salud, determinada por las elecciones que hacen las personas de las opciones disponibles acordes con las oportunidades que les ofrece su propia vida.

\section{EXCLUSIÓN/INCLUSIÓN}

¿Por qué hablar de exclusión e inclusión social? Porque vivimos en sociedades en desarrollo en las cuales existe marginación a todo nivel sobre todo entre las mujeres, las poblaciones de tercera edad y las personas discapacitadas.

La exclusión social es un fenómeno que supone la ruptura de los lazos que unen a los individuos con su sociedad, como consecuencia de una serie de procesos de marginación y desintegración social que se refuerzan mutuamente. Significa no participar de la vida económica, cultural, política del país, entre otros aspectos. Significa no pertenecer ni ser apoyado por instituciones gubernamentales o no; implica ser marginado y no tener beneficios ni responsabilidades como ciudadano en una nación.
Las variables de la exclusión social son: nivel educativo, ocupación, ingreso, salud, vivienda, programas sociales, servicios básicos de la vivienda y bienes de consumo durable, por mencionar los más importantes al ser un fenómeno multidimensional y multifactorial.

El concepto de exclusión social, según Saavedra, Ana 2001, "ha surgido principalmente en países desarrollados y ha sido utilizado para referirse al fenómeno de desintegración social, en donde se ha producido un rompimiento en las relaciones, las instituciones, en donde parte de la población está dentro y la otra está fuera".

Las principales características de la exclusión social referidas por Avaria, Andrea (2004) son:

- La exclusión se produce a través del no acceso a bienes básicos y servicios, es decir a través de formas no económicas.

- Una segunda característica estaría determinada por el acceso desigual a los mercados de trabajo y protección social.

- Una tercera característica se refiere a la exclusión de mecanismos participativos y como última característica el desigual acceso y ejercicio de derechos humanos, políticos, civiles. Es decir la exclusión social corresponde a la exclusión del mercado, instituciones sociales y culturales.

\section{SALUD}

Al introducir el vocablo salud es innegable la relación que tiene el término con el aspecto social y los estilos de vida. Si tienes acceso a los servicios de salud tus expectativas de bienestar serán mejores de lo contrario no.

Vásquez, Enrique H. et al (2003) investigadores de la Universidad del Pacífico, estudiaron los desafíos de la lucha contra la pobreza en el Perú y hallaron que las condiciones de vida de una persona serán mejores si su salud y acceso a servicios de salud se inscriben dentro de los parámetros considerados como "normales". Sin embargo, y de manera especial para un pobre 
extremo, eso no siempre sucede. De este modo, un hogar cuyos miembros gozan de buena salud y tienen además posibilidad de acceder sin mucha dificultad a los servicios de salud tendrá ventajas frente a familias que no cuentan con esa alternativa.

\section{La Salud de las mujeres}

Cuando se realizan consultas en psicología clínica es común encontrar problemáticas derivadas de la rutina en el ama de casa, la cual está sometida a presiones derivadas de su condición desigual, es la responsable de la toma de decisiones prácticamente en el hogar, es la que maneja el tema del presupuesto familiar y carga con muchas ansiedades derivadas de la crianza.

A este respecto en la experiencia que acumulé atendiendo mujeres en las especialidades de ginecología y obstetricia, psicología en consulta externa, en pediatría, etc. tuve la oportunidad de observar y atender dichas problemáticas en diferentes servicios de un hospital general de Lima metropolitana, y esto motivó mi interés por este tema.

Ya pasado el tiempo uno va elaborando ideas, centrando sus intereses y es así como quisiera abordar la salud de las mujeres desde distintos enfoques, sin embargo, en general deben ser realistas y plantear soluciones desprejuiciadas que invoquen a la libertad de decisión de las personas que buscan ayuda.

Dicho esto quisiera introducir el tema con dos autoras de habla inglesa y dos autoras españolas que curiosamente coinciden en su visión.

Ann Oakley (citado en Blesa, María Dolores 1990) refiere que, a causa del número infinito de tareas hogareñas, la libertad de un ama de casa se encontrará seriamente recortada...Algunas mujeres acuden a los médicos generales para tener entrevistas cortas con ellos, pero ellos están tan atareados que les brindarán poca atención y recibirán prescripciones que se renuevan rutinariamente en intervalos regulares.

Linda Valins (1993) sostiene que es evidente el efecto devastador de la forma en que son tratadas las enfermedades de las mujeres en las especialidades de psiquiatría y ginecología.
Pilar Blanco Prieto (1996), especialista en medicina familiar del equipo de atención primaria de San Lorenzo del Escorial hace un estudio dirigido a mujeres con dificultades socio-familiares, hiperfrecuentadoras de los centros de salud. Ella refiere el síndrome del ama de casa, sus síntomas eran dolor de cabeza o espalda, sensación de opresión o nudo en la garganta, cansancio, agotamiento, tristeza vaga.Se acometió un proyecto grupal cuyo objetivo era mejorar la salud de las mujeres, entendida como bienestar físico, psíquico y social. Para lograr tal fin era imprescindible que los médicos dieran un giro a su formación y adquirir capacidades de escucha y comunicación y ver a la persona no sólo como un conjunto de órganos y sistemas sino como un ser integrado en un medio familiar y social.

Asunción Gonzáles (1996) profesora de la Facultad de Ciencias Médicas y de la Salud de Las Palmas de Gran Canaria concluye que el sistema sanitario acaba recibiendo la demanda de descifrar un mensaje corporal que sustituye a la palabra silenciada de las mujeres. Y a éstas se les proporciona con frecuencia un diagnóstico final desconcertante. Sus dolencias son funcionales o debidas a los nervios, por lo cual, según los casos, son remitidas a los servicios de psiquiatría o se convierten en adictas crónicas a medicación psicotrópica, recetada asiduamente por los médicos de cabecera.

"Trascender esa ancestral y artificial separación cuerpo/mente que ha caracterizado históricamente toda la ciencia positivista...aún en la actualidad las ciencias médicas afirman luchar objetivamente por la salud del ser humano, pero lo hacen en un sentido exclusivamente somaticista: es decir negando la subjetividad de éste" (p. 8)

Asunción Gonzáles y Araceli Armas Santana (2000) comentan en su estudio sobre la salud de las mujeres que lo que les pasa a las mujeres tiene que ver con el modelo de identidad femenina-materna vigente, es decir con lo que se ha dado en llamar el "ideal maternal".

María Isabel Matamala (2005) se refiere al enfoque de salud y género y comenta que colectivamente hemos ido construyendo contenidos para la definición 
de salud integral, pormenorizando los aspectos que la conforman, incluidas la sexualidad y modificando la supremacía que siempre ha tenido en la tradición institucional la salud materna. Nos referimos a desmaternalizar el concepto de salud reproductiva... los derechos sexuales y reproductivos como derechos humanos básicos.

\section{LA SALUD MENTAL}

La salud mental corresponde al aspecto sano del individuo en sus interacciones armoniosas con su medio ambiente, el cual permite realizar las conductas adecuadas y necesarias para su autoafirmación y autorrealización. Por este motivo la salud mental está íntimamente ligada al desarrollo humano, tanto individual y social. Su primera consecuencia es el bienestar mental y espiritual de la persona, quien llega de esta manera a su máxima productividad cultural, intelectual y económica. Llanos, Martha (2008)

Luis Olavarría (2020) explica que la pandemia del coronavirus afectó la salud mental de unos cuatro millones de ciudadanos peruanos que sufrieron estrés y trastornos de depresión, así concluyó un informe del Instituto de Análisis y Comunicación Integración. Durante el largo confinamiento, este tipo de males aumentó en un $15 \%$.

\section{Salud Mental y Pobreza}

En América hay un elevado número de personas que aún vive en marcadas condiciones de pobreza y marginalidad, de los cuales, el grupo mayoritario está constituido por niños y niñas. Los niños pobres y sus familias están expuestos a menudo a condiciones precarias que atentan contra la salud mental y física.

En los grupos familiares pertenecientes a sectores pobres urbanos, el trabajo de producción doméstica suele ser más intenso, pues se sustituye la compra de algunos elementos por la producción casera.

En cuanto a la pobreza rural se observan situaciones que pueden afectar el bienestar de las familias, tales como una menor disponibilidad de los servicios básicos como agua potable y gas. La distancia entre los hogares campesinos y la lejanía de los centros poblados, favorece el aislamiento de las familias, dificulta la realización de trámites, compras y el acceso a los servicios de salud. Kotliarenco, María Angélica, Cáceres, Emma et al. (2001)

Las mujeres son las más pobres entre los pobres. El círculo de la pobreza las envuelve con un abrazo más apretado cuanto más desprotegidas se encuentran en sus sociedades.

La pobreza en el mundo afecta sobre todo a las mujeres. La mayoría de los 1500 millones de personas que viven con 1 dólar o menos al día son mujeres. Y la brecha que separa a los hombres de las mujeres atrapadas en el ciclo de la pobreza ha seguido ampliándose en el último decenio. Este fenómeno ha llegado a conocerse como "la feminización de la pobreza"

Tradicionalmente, en la mayoría de las sociedades, ha sido la mujer la que ha cargado con el peso de la economía familiar. El trabajo doméstico suele correr de su cuenta, pero no se recompensa económicamente ni se reconoce su trabajo en este ámbito. Solidaridad Internacional(2006)

\section{Participación de la Mujer en Salud}

Las mujeres tienen una gran participación en el ámbito sanitario, desde el área de limpieza, las profesionales de la salud, incluso actualmente en las áreas de gestión. Sin embargo, aún se escucha comentarios que menoscaban el trabajo de las mismas, o de su actuación en las tareas profesionales.

Carme Valls Llobet, (1996) comenta que hay que hacer visibles las diferencias en la Salud y la Enfermedad entre mujeres y hombres, erradicar el sesgo androcéntrico en la investigación científica. Temas importantes son: la doble jornada y salud de las mujeres; el estrés, sus causas y sus consecuencias en la salud; ergonomía y salud laboral; organización del trabajo y salud mental; trabajo y acoso sexual; la importancia de la metodología de la investigación; el reparto de las tareas domésticas, o el abuso de psicofármacos.

María Isabel Matamala, (1996) es una estudiosa del tema de la mujer en Chile y América Latina y hace una reflexión sobre el rol de las mujeres en la Sanidad 
pública. Las mujeres que trabajan en el área de Sanidad soportan una carga horaria de trabajo semejante a las ramas de la industria. En el sector salud, a la doble jornada se agrega la carga psicológica que supone el interactuar con personas demandantes en lo referido a vida/muerte y salud/enfermedad. Su socialización condiciona a estas mujeres para acoger y hacerse cargo de los problemas y necesidades de otros/as. No obstante, las condiciones de trabajo y el peso de su carga horaria dificultan la realización del internalizado mensaje, ser para otros/as sobre todo en los estratos inferiores, que soportan presiones en un contexto de mayor precariedad y opresión.

El desgaste físico y emocional de estas jornadas, en las cuales está como telón de fondo la división de las mujeres entre sus mundos público y privado, explica desde una mirada de género lo que sucede con la salud y las relaciones interpersonales de las mujeres del sector. La tensión e irritabilidad, así como los estados crónicos de fatiga física y mental, están en la base de sus interacciones. La fatiga aparece en sus niveles crónico y patológico, ya que los malestares señalados reflejan impacto orgánico, alteraciones del sueño y del humor.

María Isabel Matamala (2005) menciona que, para acabar con la inequidad en el concepto de salud, implica transformaciones desde el ámbito de las ciencias económicas hasta transformaciones en gestión así como en participación en salud, con incorporación verdadera de la comunidad, particularmente las mujeres en las decisiones y evaluaciones.

\section{CONCLUSIONES}

1. La salud y el bienestar son conceptos que se relacionan entre sí. El bienestar alude a las actitudes y comportamientos que mejoran la calidad de vida de las personas y nos ayudan a llegar a un estado de salud óptimo.

2. La forma en que vivimos constituye los estilos de vida. Estos, si son saludables implican el conocer aquellos comportamientos que pueden mejorar o socavar la salud de los individuos.
3. La salud mental corresponde al aspecto sano del individuo en sus interacciones armoniosas con su medio ambiente.

4. Es evidente que es necesario mejorar la salud de las mujeres, entendida como un estado de bienestar físico, psíquico y social.

5. Si se estudia el tema de la salud es imprescindible que los médicos den un giro a su formación y adquieran capacidades de escucha y comunicación para ver a la persona no como un conjunto de sistemas y órganos sino como un ser integrado en un medio familiar y social.

\section{DEFINICIÓN DE TÉRMINOS}

Bienestar: Nivel de vida de un grupo de personas

Estilo de Vida: calidad de vida

Equidad: cualidad de las acciones en que se trata a cada uno como se merece o como le corresponde y de las personas que actúan de ese modo

Exclusión Social: es un fenómeno que supone la ruptura de los lazos que unen a los individuos con su sociedad, como consecuencia de una serie de procesos de marginación y desintegración social que se refuerzan mutuamente

Género: Es la construcción cultural que asigne diferentes conductas, actividades y funciones a las mujeres y los hombres, a las chicas y los chicos, a las niñas y los niños. Incluye las características que se atribuyen a la feminidad y masculinidad

Salud: ausencia de afecciones o enfermedades y como un estado de bienestar integral

Salud mental: Presencia de bienestar físico y emocional

Somaticista: dícese de cualquier afección que tenga base orgánica. Se contrapone a funcional. 


\section{REFERENCIAS}

Arboix, M. (2013, Mayo/19). Estilo de vida y salud. http://www.consumer.es/salud/estilo-de-vida-ysalud.html

Blanco Prieto, P. (1996). Nuestra salud física, psíquica y social. Mujeres, $24,4^{\circ}$ trimestre, p. 10.

Blesa, M. D. (1990). Las Actitudes de las Madres Cesareadas frente a la relación madre-niño. Tesis de Magíster en Psicología. Universidad Femenina del Sagrado Corazón.

Fischman, D. (2010). La alta rentabilidad de la felicidad. Universidad Peruana de Ciencias Aplicadas.

Gonzáles, A. (1996). A la Búsqueda de una nueva relación entre la medicina y las mujeres. Mujeres, $24,4^{\circ}$ trimestre, pp. $8-9$

Gonzáles, A.,Armas Santana,A. (2000). Aproximación a la salud de las mujeres desde una perspectiva de género (i)Web Especial Canarias/Salud $\mathrm{y}$ Género. http:// fedsp.org/ ab47510/documentos/ salud2000/148/aprox36-38-148.pdf

Kotliarenco, M. A., Cáceres, E. et al. (2001). La pobreza desde la mirada de la resiliencia. Revista Peruana de Psicología, 6, 6 (11), pp 69-78.

Lamas Rojas, H., Murrugarra A., A., Márquez A., Lamas, F., Silva F. P., Cortés, O. S., Arévalo, C., Alvárez, J. E., Avaria, A. (2004). Maltrato al adulto Mayor. Discapacidad y Exclusión: Otra forma de Violencia. Colección de Materiales y Textos. Colegio de Psicólogos del Perú.

Lopategui Corsino, Edgar (2001). Salud y Bienestar. http://www.saludmed.com

Llanos, M. (2008). Bioética y Desarrollo Humano para una cultura de Paz. Avances en Psicología, 16 (1), p. 40.

Matamala, M. I. (1996). Mujeres, género y trabajo en salud. Mujeres, 23, $3^{\circ}$ trimestre, pp. 8-9.
Matamala, M.I. (2005). Tenemos que democratizar el conocimiento que vamos construyendo entre todas. CEME Web productions 2005. http://www. archivo-chile.com

Merino, B. (1998). La mujer: talento humano, rompiendo barreras. Avances en Psicología, 13(1), pp. 23-32.

Oblitas, L. (2008). Psicología de la Salud: Una ciencia del bienestar y la felicidad. Avances en Psicología, 16 (1) pp. 17-22.

Olavarría, L. (2020, Julio, 13). Encierro afectó salud mental de población. Unos cuatro millones con estrés y depresión. Diario Correo/Redacción, p.4

Oliver, A., Ruiz, M. J. (2006). Mujeres y pobreza. Crónica de un debate en Nueva York. Red europea de mujeres periodistas. Informe Beijing +10 Emakunde, 60, pp. 14-15.

Saavedra, A. (2001). Discapacidad: exclusión / inclusión. Revista Mad (5). https://revistamad. uchile.cl/index.php/RMAD/article/view/14826

Solidaridad Internacional (2006). La feminización de la pobreza. Monográfico Mujeres y Desarrollo, 45 , pp. 4-5-8.

Valins, L. (1993). Intimate matters. Restoring balance and harmony to the feminine experience. Gaia Books Limited.

Valls Llobet, C., Messing, K., Lundberg, U., Dean, K., Kaupinnen, K. (1996). Hacer visibles las diferencias. Mujeres, 22, $2^{\circ}$ trimestre, pp. 8-9.

Vásquez, E., Aramburú, L., C., Figueroa, C., Parodi, C. (2003). Los desafíos de la lucha contra la pobreza extrema en el Perú. Universidad del Pacífico. Centro de Investigación.

Fecha de recepción: 30 de junio 2020

Fecha de aceptación: 24 de setiembre 2020 